\title{
Perfusion Paths: Inference of Voxelwise Blood Flow Trajectories in CT Perfusion
}

\author{
David Robben ${ }^{1}$, Stefan Sunaert ${ }^{2}$, Vincent Thijs ${ }^{3}$, Guy Wilms ${ }^{2}$, Frederik \\ Maes $^{1}$, and Paul Suetens ${ }^{1}$ \\ 1 iMinds - Medical Image Computing (ESAT/PSI), KU Leuven, Belgium \\ 2 Department of Radiology, University Hospitals Leuven, KU Leuven, Belgium \\ 3 Department of Neurology, University Hospitals Leuven, KU Leuven, Belgium
}

\begin{abstract}
In CT perfusion imaging (CTP) multiple, consecutive 3D CT scans of an organ are made during the administration of contrast agent. This results in a $3 \mathrm{D}$ movie of the contrast agent entering and subsequently leaving the organ. Currently, this modality is mainly used for voxelwise analysis of perfusion parameters such as blood flow, blood volume, transit time etc. In this work, we propose to analyze these images in a more global fashion and introduce a method to infer the connectivity of the vascular structure underlying the perfusion - even if the vasculature itself is on a subvoxel scale. This novel approach enables several new applications for CTP. The feasibility of the method is illustrated on clinical data.
\end{abstract}

\section{Introduction}

The vascular system plays a crucial function in our body, permitting blood to circulate from the heart, through arteries and arterioles to the capillary bed, and through venules and veins back to the heart. In the capillary bed, perfusion takes places: the process of exchanging nutrients, oxygen, waste, etc. with the surrounding cells. Perfusion is essential for the function and survival of living tissue and hence there is vasculature virtually everywhere in the body. Traditional vascular imaging modalities, such as CT angiography (CTA) and MR angiography (MRA), have a limited imaging resolution. In the resulting image, the vascular structure is sparse and most voxels will be considered non-vessel, while in reality they do contain (micro)vasculature.

In CT perfusion imaging (CTP) multiple, consecutive 3D CT scans of an organ are made during the intravenous administration of a iodine contrast agent bolus. Each scan is a volumetric image, showing for every voxel the X-ray attenuation coefficient in Hounsfield units (HU). The result is a 3D movie of the contrast agent entering and subsequently leaving the organ. Alternatively, it can be considered as a 3D image with in every voxel a time series of the HU variation due to the local contrast passage. Subtracting the first image, which is made

\footnotetext{
* David Robben is supported by a Ph.D. fellowship of the Research Foundation Flanders (FWO).
} 

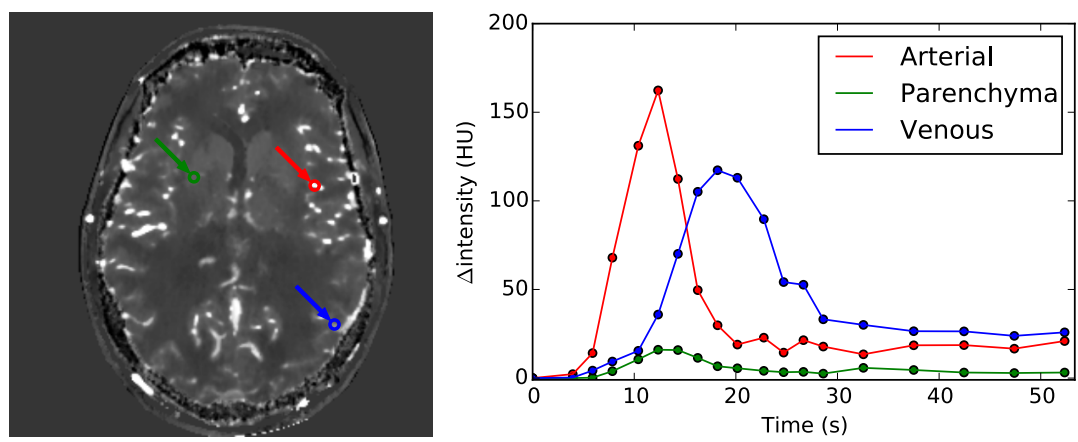

Fig. 1. Illustration of a cerebral CT perfusion. Left: axial slice of a cerebral CTP during the late arterial phase. The red, blue and green markers are located in respectively an artery, a vein and the grey matter. Right: time series in the markers.

before the contrast agent enters the organ, from the subsequent ones results in difference images that show the increase in $\mathrm{HU}$ due to the contrast agent. An illustration of a cerebral CTP image is given in Fig. 1.

This modality is very information rich and useful in clinical practice. First, each image of the movie can be considered a traditional CT angiography (CTA), thus allowing assessment of the vasculature in both arterial and venous phase images. More important, however, is the voxelwise calculation of clinically relevant parameters such as time to peak, mean transit time, blood flow and blood volume [8]. These voxelwise parameter maps are used for the diagnosis of stroke - a.o. for the assessment of the tissue at risk - and help making therapeutic decisions. Organs of interest include liver, lungs and brain, with the latter being the most important and also the focus of this work.

Although these voxelwise summary measures prove to be very useful in clinical practice, additional information may be deduced from CTP, especially regarding the vascular structure underlying the perfusion and the trajectories the blood follows throughout the organ. In this work, we propose a method to deduct the vascular connectivity throughout the organ - even if the vasculature itself is on a subvoxel scale. To the best of our knowledge, there is no other modality or image analysis technique that can infer this information, which has a wide range of potential applications. In clinical practice, it is relevant to determine the perfusion territories of arteries (i.e. the regions supplied by those arteries). There are atlases that describe the perfusion territories of the major arteries, but as there is considerable inter-subject variability, it is beneficial to measure them in vivo for each patient specifically [7]. With knowledge of the complete vascular connectivity, calculation of the perfusion territory of an arbitrary artery becomes possible. In research, one might want to investigate the inter-subject variability in vascular connectivity, how this changes during a stroke or whether there is correlation with incidence and outcome of vascular pathology.

In the following sections we first discuss related works, then present our method, and finally illustrate the feasibility of the method on clinical data. 


\section{Related work}

Currently, we can distinguish five techniques to image the perfusion territories.

In catheter-based angiography, an artery is selectively injected with contrast agent through a catheter and subsequently the region of interest perfused by this artery is imaged. Although this method gives excellent image quality, it is also very invasive and time consuming.

Regional arterial spin labeling is an MRI technique where arterial blood is selectively magnetized (labeled) and the magnetic disturbance of this labeled blood is imaged in the region of interest. This technique has several advantages, such as the ability to detect overlapping perfusion territories, the use of nonionizing radiation and its non-invasive nature. On the other hand, there is very weak signal inside the gray matter, acquisition is time consuming and every additional artery for which the perfusion territory needs to be measured, demands more time.

Then, there is $C T$ and MR perfusion. To the best of our knowledge, the only existing works regarding perfusion territory determination in those modalities are those of Christensen et al. The first work [3] describes a bolus tracking method for perfusion MRI. First, the arrival time of the bolus is calculated for every voxel in the image. The authors reason that the arrival times are monotonically rising from the point where the blood enters the image, along the vascular trajectories and towards the periphery. Hence, for every voxel, the least descending path is calculated, i.e. the path that visits each time the neighboring voxel with the highest arrival time that is lower than the arrival time of the current voxel. Such a path always ends in a local minimum of the arrival time, which they assume to be an arterial source along which blood enters the imaged region. They demonstrate the method on a single axial slice. In a second work $[1,2]$, a method is presented for perfusion CT. The authors argue that, while the assumption of monotonically rising arrival times is true in theory, the high blood velocity in big arteries $(50 \mathrm{~cm} / \mathrm{s})$, the relatively low sampling frequency (1 image/s) and the small voxel size (less than $1 \mathrm{~mm}^{3}$ ) make their estimation of the arrival time too noisy for the previous method to work: the many local minima in the arrival time will result in as many incorrect arterial sources. Instead they propose using competitive region growing on the arrival time image: starting from several anatomically labeled seed regions, region growing with a low threshold is applied, the selected voxels are added to their respective seed regions, the threshold is increased and the process is repeated. This is demonstrated on cerebral CTP images, where manual segmentations of the anterior, middle and posterior arterial trees are used as seeds and their method subsequently finds the associated perfusion territories.

Finally, there is the possibility of extrapolation of the vascular image. Selle et al. [6] predict the vascular territories of the liver by segmenting the hepatic arteries, anatomically labeling them and assigning each non-vessel voxel to its closest artery. 


\section{Mathematical model}

With a perfect, unlimited resolution scanner, modeling vascular connectivity would boil down to modeling the organ's microvasculature. But since our scanner's resolution is two magnitudes larger (a capillary has a diameter of $5 \mu \mathrm{m}$ ), this is impossible. Instead we propose to model the connectivity of the underlying vasculature as the connectivity of the voxels in the PCT image $I$.

This (now unknown) connectivity can be represented as a directed graph $\mathrm{G}=(\mathrm{V}, \mathrm{E})$. Every voxel $v_{i}$ in the image $I$ is a vertex: $v_{i} \in V$. If there is blood flow between neighbouring voxels $v_{i}$ and $v_{j}\left(v_{j} \in \mathcal{N}\left(v_{i}\right)\right.$, using 26-connectivity), there is an edge $e_{i j}=\left(v_{i}, v_{j}\right) \in E$. We propose to model the arterial and venous connections separately. In this work, we further only consider the arterial case, but as discussed later, the same approach could be used for the veins.

Now, finding the vascular connectivity of an image, boils down to finding this unknown set of edges $E$. We want to find the most probable set:

$$
E^{*}=\operatorname{argmax}_{E \in \mathbb{E}} \log P(E \mid I)
$$

where $\mathbb{E}=\{E\}$ is the set of acceptable edge sets. Let $V_{s} \subset V$ be the set of arterial voxels on the image border; these are the sources of blood in the imaged region. An edge set $E$ is acceptable if every perfused voxel is reachable from a source $v_{s} \in V_{s}$ and the only perfused voxels without an incoming edge are those in $V_{s}$.

Finally, we do not consider vascular anastomoses: these are connections between two vascular trees, such as present in the Circle of Willis. If there is an anastomosis, only one connection will be modeled. This approximation reduces the problem complexity greatly: $G$ contains now only tree-like structures. Or equivalently, every voxel $v_{i}$ has a single path from a source $v_{s} \in V_{s}$, where a path is a tuple of neighboring voxels: $\operatorname{path}\left(v_{i}\right)=\left(v_{s}, \ldots, v_{j}, \ldots, v_{i}\right)$. Additionally, for every other voxel $v_{j}$ on that path it holds that $\operatorname{path}\left(v_{j}\right) \subset \operatorname{path}\left(v_{i}\right)$. We propose to call them perfusion paths: the voxel paths the blood appears to follow from the arterial sources towards each perfusion site. If those perfusion paths are known, the edges are also known: $E=\left\{\left(\operatorname{path}\left(v_{i}\right)_{j}, \operatorname{path}\left(v_{i}\right)_{j+1}\right) \mid v_{i} \in V, 1 \leq\right.$ $j \leq\left(\mid\right.$ path $\left.\left.\left(v_{i}\right) \mid-1\right)\right\}$. Also, it is now easy to determine for every voxel $v_{i}$ its perfusion territory $P T\left(v_{i}\right)$, the set of voxels that is perfused through this voxel: $P T\left(v_{i}\right)=\left\{v_{j} \mid v_{i} \in \operatorname{path}\left(v_{j}\right)\right\}$.

We propose to find those perfusion paths by tracking the contrast bolus throughout the imaged volume. We can find those perfusion paths by minimizing for every voxel the cost of its path to a source independently. For a wide range of cost functions, this can be done efficiently with dynamic programming:

$$
\begin{aligned}
& \operatorname{path}\left(v_{i}\right)= \begin{cases}\left(v_{i}\right), & \text { if } v_{i} \in V_{s} \\
\arg \min _{p \in \mathcal{P}_{i}}(\operatorname{cost}(p)), & \text { otherwise }\end{cases} \\
& \mathcal{P}_{i}=\left\{\operatorname{path}\left(v_{j}\right)+\left(v_{i}\right) \mid v_{j} \in \mathcal{N}\left(v_{i}\right)\right\}
\end{aligned}
$$


Following the idea that the bolus arrival times should be monotonically rising from the arterial source towards the peripheral regions, we use:

$$
\operatorname{cost}(p)=\max _{v_{j} \in p} \operatorname{arrival}\left(v_{j}\right)
$$

with arrival giving the arrival time of the contrast bolus in that voxel. In Eq. 2, if a voxel is reached by several paths with the same cost, the path $p$ whose second last vertex $p_{|p|-1}$ has the lowest $\operatorname{cost} \operatorname{cost}\left(\operatorname{path}\left(p_{|p|-1}\right)\right)$ will be taken.

The approach of Selle et al. [6] can also be described in our model and will be used for evaluation purposes. Its cost function is:

$$
\operatorname{cost}(p)=\sum_{2 \leq j \leq|p|} \operatorname{dist}\left(p_{j-1}, p_{j}\right)\left(1-A\left(p_{j}\right)\right)
$$

with $A$ a binary arterial segmentation and dist giving the Euclidean distance between a pair of voxels.

Arrival time estimation Our method requires a very accurate estimation of the arrival time. Christensen et al. [3] describe several methods to estimate the arrival time and conclude after simulations that the first moment of the time series is the best metric. However, due to recirculation of the contrast agent, this metric will be heavily influenced by the tail of the timeseries (see Fig. 1) and overestimate the arrival time by a variable factor depending on the length of the tail. We propose to use the time to half $\max$ (THM), whose determination is better conditioned than the frequently used time to peak (TTP). Intuitively: the time series is much flatter at the maximum than at its half maximum, making determination of the exact time when the maximum is reached much more susceptible to noise. A formal proof is given in the supplementary material ${ }^{1}$.

\section{Experiments}

There is no ground truth method for measuring the blood flow trajectories in vivo. Instead, we qualitatively evaluate our method by comparison with medical literature, demonstrate its feasibility and compare it with alternatives. We use four clinical cerebral CTP datasets from one healthy, one tumor and two stroke patients. Each dataset is acquired on a Toshiba Acquilion One, has 19 time points and has a voxel size of $0.443 \times 0.443 \times 0.5 \mathrm{~mm}^{3}$, resampled to $0.886 \mathrm{x} 0.886 \times 1.0 \mathrm{~mm}^{3}$ to speed up calculation and reduce noise. First, motion correction is performed: every image $I_{t}$ with $t>0$ is rigidly aligned to the first image $I_{0}$ using the Elastix Toolbox [4]. Noise is suppressed through both spatial and temporal filtering. Spatial filtering is done by bilateral Gaussian smoothing on the time series, called TIPS [5], with a $\sigma$ of $4 \mathrm{~mm}$. Temporal smoothing is done with a $\sigma$ of 1 image. Finally, the images are processed with the proposed method.

\footnotetext{
${ }^{1}$ See www.medicalimagingcenter.be/public/MIC/publications/DR_MICCAI2015
} 


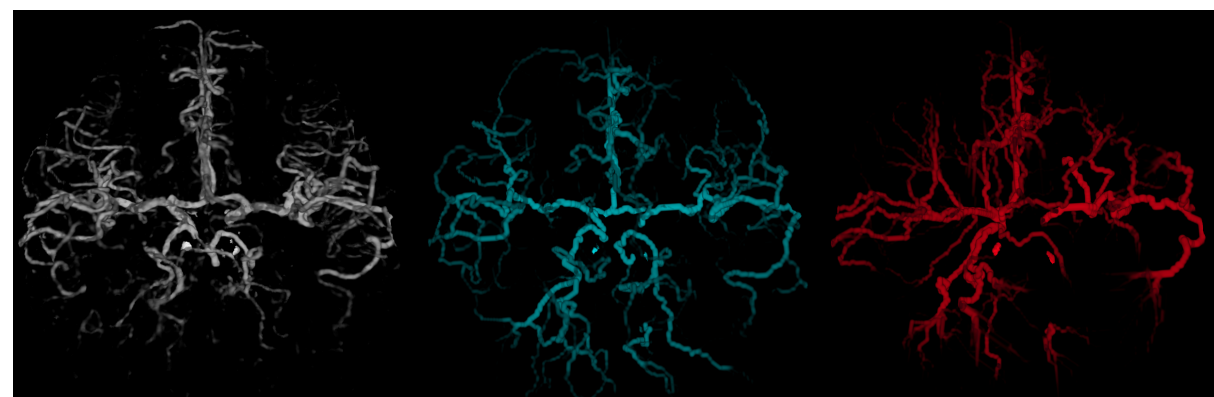

Fig. 2. Relation between the contrast enhancement during the arterial phase and perfusion territory volume (PTV). Three dimensional renderings of a subtraction image during the arterial phase (in grey) and of the logarithm of the PTV calculated using the proposed THM (in blue) and TTP (in red).

Perfusion territory volume Intuitively, one would expect that voxels in the big arteries have large perfusion territories and vice versa. Hereto, we compare for the cerebral voxels their contrast enhancement during the arterial phase with their perfusion territory volume (PTV), the volume that is perfused through this voxel: $\left|P T\left(v_{i}\right)\right|$. Fig. 2 contains results obtained by our method using either THM or TTP for arrival estimation, showing that the proposed metric outperforms the latter. Even though the perfusion paths are calculated without knowledge of the amount of contrast enhancement, the results show that this relation indeed holds, suggesting that the perfusion paths are realistic and indeed follow the arteries. Nevertheless, there are places where the perfusion paths take 'shortcuts': it is for example impossible to distinguish the left and right anterior cerebral artery. For the four datasets, the correlations between the intensity and the logarithm of the PTV using TTP are: $0.15,0.12,0.13$ and 0.14 ; using THM yields: 0.27 , $0.20,0.26$ and 0.27 . Visualizations can be found in the supplementary material.

Perfusion territories of MCA, ACA and PCA We manually place markers on the M1-segments, the A2-segments and P2-segments and visualize their perfusion territories using the proposed method and the method of Selle et al. [6]. Results for the healthy subject are shown in Fig. 3. Even though the boundaries produced by the proposed method are more crotchety, the results are more in concordance with the medical literature: the posterior inferior part of the brain is supplied by the PCAs, there is symmetry between the left and right PCA, and the superior posterior part of the brain is supplied by the ACA [7]. Furthermore, we speculate that in case of vascular pathology, such as stenosis, prediction of the territories based on the vasculature becomes even more unreliable. More examples and movies can be found in the supplementary material.

Perfusion territory of the lenticulostriate performators It is described that the lenticulostriate perforators, which branch off from the A1 and M1 seg- 


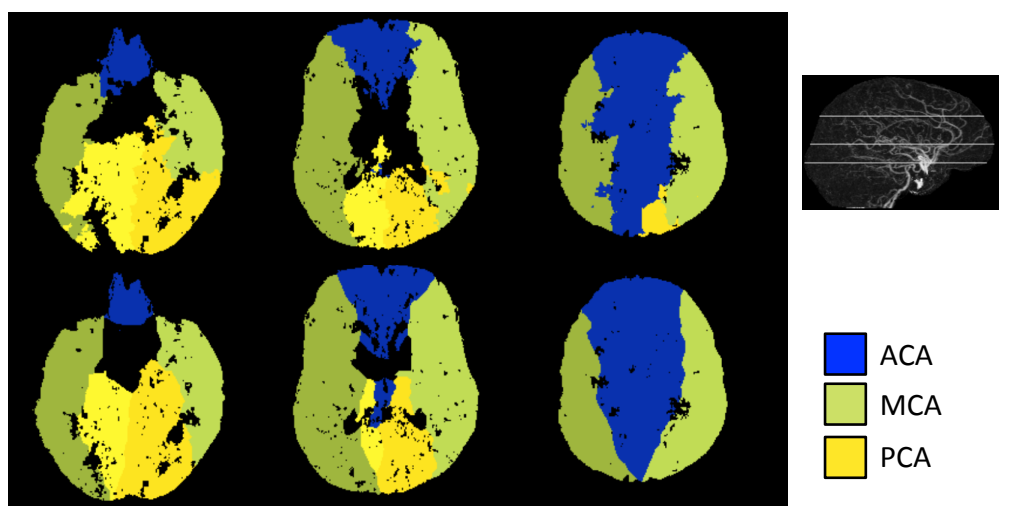

Fig. 3. The perfusion territories of the anterior cerebral arteries (ACAs), the left and right middle cerebral arteries (MCAs) and the left and right posterior cerebral arteries (PCAs). Three axial slices are shown in a healthy subject, the top row calculated by the proposed method, the bottom row by method of Selle et al. [6]. Note that, even though the boundaries produced by the proposed method are more crotchety, they are more in concordance with the medical literature (see text).

ment, provide blood to the putamen [7]. To validate our method, we segment the putamen and calculate the volumetric fraction that is indeed perfused from the M1 and A1 segment. We find that the fraction of putamen volume perfused by the lenticulostriate perforators is in the four datasets: $0.70,0.80,0.57$ and 0.93. In comparison, the method of Selle et al. [6] finds 0.31, 0.49, 0.38 and 0.56, which is significantly lower.

\section{Discussion and conclusion}

We presented a method to infer the arterial perfusion paths. We showed the plausibility of the achieved results and demonstrated its advantage over existing methods.

From a methodological point of view, our formulation is a generalization of the works of Christensen et al. We follow the contrast flow like in [3], but due to the improved formulation and careful estimation of the arrival times, we did what they deemed impossible: trace the blood flow trajectories back to the arterial sources. The main advantage of our method over the competitive region growing approach of [1], is the ability to determine the territory of any single artery or even voxel, without prior segmentation of all 'competing' arteries or voxels.

The same formulation could be applied to infer the venous trajectories. Hereto, one should invert the modeling: the venous trees are rooted in the contrast sinks i.e. the venous voxels on the image border and there is a directed edge $e_{i j}$ if voxel $v_{i}$ receives venous blood from voxel $v_{j}$. The cost function is 
now in terms of the contrast departure time, which is in theory monotonically decreasing from the contrast sinks. As such, $\operatorname{cost}(p)=\max _{v_{j} \in p}-\operatorname{departure}\left(v_{j}\right)$.

The proposed cost function bears close resemblance to doing a watershed segmentation on the arrival time image, with the extension that the flow path of the water is stored. Currently, we only use the arrival time for inferring the vascular trajectories. It is however possible to include extra information, e.g. the maximum increase in intensity or even the full time series. Additionally, anatomical knowledge about the vascular connectivity can be included: e.g. there is no arterial connectivity between the two hemispheres. This can be enforced by excluding edges $\left(v_{i}, v_{j}\right)$ with $v_{i}$ and $v_{j}$ in different hemispheres from the acceptable edge sets. Dynamic programming can naturally handle such constraints.

In future work, we will investigate more elaborate cost functions including intensity information and anatomical knowledge. Additionally, the method will be compared with regional arterial spin labeling to assess its accuracy in determining the perfusion territories.

Acknowledgement We would like to thank Walter Coudyzer for the collection of the images.

\section{References}

1. Christensen, S.: Identification of vascular territories (WO 2013/159147A1) (2012)

2. Christensen, S., Brien, B.O., Menon, B., Bivard, A., Campbell, B.: Mapping of Cerebral Vascular Territories Using Whole Brain Perfusion CT Imaging : A New Method. In: International Stroke Conference. vol. Acute Neur, p. Abstract 54 (2012)

3. Christensen, S., Calamante, F., Hjort, N., Wu, O., Blankholm, A.D., Desmond, P., Davis, S., Østergaard, L.: Inferring origin of vascular supply from tracer arrival timing patterns using bolus tracking MRI. Journal of Magnetic Resonance Imaging 27, 1371-1381 (2008)

4. Klein, S., Staring, M., Murphy, K., Viergever, M.A., Pluim, J.P.W.: Elastix: A toolbox for intensity-based medical image registration. IEEE Transactions on Medical Imaging 29(1), 196-205 (Jan 2010)

5. Mendrik, A.M., Vonken, E.j., van Ginneken, B., de Jong, H.W., Riordan, A., van Seeters, T., Smit, E.J., Viergever, M.A., Prokop, M.: TIPS bilateral noise reduction in 4D CT perfusion scans produces high-quality cerebral blood flow maps. Physics in medicine and biology 56, 3857-3872 (2011)

6. Selle, D., Preim, B., Schenk, A., Peitgen, H.O.: Analysis of vasculature for liver surgical planning. IEEE Transactions on Medical Imaging 21(11), 1344-1357 (Nov 2002)

7. Tatu, L., Moulin, T., Bogousslavsky, J., Duvernoy, H.: Arterial territories of the human brain. Neurology 50(6), 1699-1708 (1998)

8. Wintermark, M., Maeder, P., Thiran, J.P., Schnyder, P., Meuli, R.: Quantitative assessment of regional cerebral blood flows by perfusion CT studies at low injection rates: a critical review of the underlying theoretical models. European radiology 11(7), 1220-30 (Jan 2001) 